\title{
Impacto de uma metodologia interativa de ergonomia de conscientização
}

\author{
Impact of an interactive methodology for ergonomics awareness \\ Impacto de una metodología interactiva de ergonomia de concientización \\ Joyce Ribeiro Rothstein', Angélia Berndt² , João Carlos de Souza Moraes ${ }^{3}$, Fábio Juner Lanferdini ${ }^{4}$
}

RESUMO I A ergonomia tem demonstrado avanços ao ser pró-ativa com a concepção de ambientes ergonomicamente adequados e com a conscientização do uso correto dos postos de trabalho. Nesse contexto, destaca-se o papel da ergonomia de conscientização, que confere ao indivíduo papel central no processo de educação em saúde. O presente estudo teve como objetivo avaliar o impacto de uma metodologia interativa de ergonomia de conscientização no conhecimento de trabalhadores de uma indústria têxtil. A amostra foi selecionada a partir do parecer do comitê de ergonomia da empresa. Participaram do estudo 328 trabalhadores (idade: 33,94 99,15 anos). Foram aplicadas intervenções interativas nos temas: Corpo Humano, Visão, Audição, Coluna vertebral, Membros Superiores e Membros Inferiores. O impacto da metodologia foi mensurado com a aplicação de um questionário criado e validado para o estudo. Para a comparação da pontuação antes e após as intervenções foi utilizado o teste $t$ de Student (pareado), analisado no pacote estatístico SPSS 19.0 para Windows com significância de $p<0,05$. As questões qualitativas foram categorizadas, e a frequência das informações constatada. Observou-se aumento na pontuação total do questionário $(8,07 \pm 1,03$ para 8,76 $\pm 0,50$; $p<0,001$ ), o que demostra que o programa teve impacto na aquisição de conhecimento por parte dos trabalhadores em relação a segurança e saúde.

Descritores I engenharia humana; saúde do trabalhador; conhecimento; indústria têxtil.
ABSTRACT I Ergonomics has demonstrated advancements being proactive in the design of ergonomically suitable environments and promoting awareness of the correct use of the workplace. In such context, ergonomics' role on awareness raising is highlighted, since it provides the individual with a central role in the health education process. The purpose of this study was to assess the impact of an interactive program of awareness raising Ergonomics in relation to safety and health knowledge of workers of a textile factory. The sample was selected from a program initiated by the Ergonomics Committee of the company. 328 workers took part in the study (aged 33.94 \pm 9.15 ). Interactive and brief interventions on the following topics were applied: Human Body, Vision, Hearing, Human Movement, Spine, Upper and Lower Limbs. The impact was measured by means of a questionnaire that was created and validated for this study. For the comparison of the score Pre and Post intervention, a paired Student's t-test was used and analyzed in SPSS 19.0 for Windows with a significance of $p<0.05$. Qualitative questions were categorized and the frequency of information was verified. There was an increase in the total questionnaire score $(8.07 \pm 1.03$ to 8.76 $\pm 0.50 ; p<0.001$ ), which demonstrates that the program had a significant impact on workers' knowledge acquisition in relation to health and safety.

Keywords I human engineering; occupational health; knowledge; textile industry.

Estudo desenvolvido no Serviço Social da Indústria de Santa Catarina - SESI (DR) - Florianópolis (SC), Brasil.

'Fisioterapeuta, Mestre em Ciências do Movimento Humano; Consultora do SESI/SC, Gestora do Projeto Ergonomia de

Conscientização - Florianópolis (SC), Brasil.

${ }^{2}$ Doutora em Engenharia de Produção; Coordenadora do Centro de Tecnologia do Social do SESI/SC - Florianópolis (SC), Brasil.

${ }^{3}$ Mestre em Engenharia Ambiental; Engenheiro Civil e de Segurança do Trabalho da Marisol S.A., Professor Universitário da Associação Franciscana de Ensino Senhor Bom Jesus - Blumenau (SC), Brasil.

${ }^{4}$ Educador Físico; Mestre em Ciências do Movimento Humano - Florianópolis (SC), Brasil. 
RESUMEN I La Ergonomía tiene demostrado avances al ser proactiva con la concepción de ambientes ergonómicamente adecuados y con la concientización del uso correcto de los puestos de trabajo. En éste contexto, se destaca el papel de la ergonomía de concientización, que confiere al individuo el papel central en el proceso de educación en salud. El presente estudio tiene como objetivo evaluar el impacto de una metodología interactiva de ergonomía de concientización en el conocimiento de trabajadores de una misma industria textil. La muestra fue seleccionada a partir de la opinión del comité de ergonomía de la empresa. Participaron del estudio 328 trabajadores (Edad: 33,94ะ9,15 años). Fueron aplicadas educaciones interactivas en los temas: Cuerpo Humano, Visión, Audición, Columna Vertebral, Miembros
Superiores y Miembros Inferiores. El impacto de la metodología fue medido con la aplicación de un cuestionario creado y validado para el estudio. Para la comparación de la puntuación Pre y Post intervenciones fue utilizado el Test $t$ de Student (pareado), analizado en el paquete estadístico SPSS 19.0 para Windows con significancia de $p<0,05$. Las preguntas cualitativas fueron categorizadas y la frecuencia de las informaciones constatadas. Se observó aumento en la puntuación total del cuestionario (8,07×1,03 para 8,76 $\pm 0,50 ; p<0,001$ ), el que demostró que el programa tiene impacto en la adquisición del conocimiento por parte de los trabajadores en relación con la seguridad y salud.

Palabras clave I ergonomía; salud del trabajador; conocimiento; industria textil.

\section{INTRODUÇÃO}

O Brasil possui a maior taxa de doenças ocupacionais e acidentes de trabalho da América Latina ${ }^{1}$, responsáveis por elevados custos previdenciários e pela redução da produtividade ${ }^{2-5}$.

Uma das formas de prevenir doenças ocupacionais é a adoção de práticas ergonômicas ${ }^{6-9}$. Bom Sucesso ${ }^{10}$ relata que a prática ergonômica é essencial para o sucesso da empresa por impactar na qualidade de vida do trabalhador. A literatura tem apontado que a maioria dos programas de ergonomia oferecidos pelas empresas é voltada para a ergonomia de correção e de concepção. Em síntese, visam conceber e planejar o ambiente de trabalho, respeitando o fluxo de produção e as características físicas do indivíduo, bem como fazer adequações no posto de trabalho, quando necessário ${ }^{11,12}$.

A ergonomia de conscientização ${ }^{13}$ vem complementar a ergonomia de concepção e correção, pois, por meio de treinamentos, o trabalhador poderá aprender a forma adequada de utilizar seu ambiente de trabalho, cuidar do corpo ao adotar posturas corretas para realizar suas atividades, bem como usar equipamentos de proteção, entre outras medidas necessárias para a prevenção de doenças e acidentes. Enquanto a ergonomia de correção e concepção tem como foco principal as alterações no ambiente de trabalho, a ergonomia de conscientização traz à tona a importância de ter o indivíduo como foco central e a necessidade de conscientizá-lo ${ }^{13,14}$.

É possível verificar na literatura a descrição de programas que envolvem a ergonomia de conscientização $0^{4,7111,13,15}$, contudo, as intervenções descritas geralmente são pontuais e informativas, como palestras ou aulas expositivas dialogadas, e o trabalhador é mero espectador, o que suscita a necessidade de intervenções interativas e diferenciadas, inseridas em metodologia estruturada, na qual o trabalhador é agente ativo na construção do conhecimento, com papel central no processo de promoção da saúde.

Com base nos fatores citados, o presente estudo teve como objetivo avaliar o impacto de uma metodologia interativa de ergonomia de conscientização no conhecimento dos trabalhadores de uma indústria têxtil.

\section{METODOLOGIA}

A presente pesquisa caracteriza-se como uma pesquisa pré-experimental, pois visou investigar a relação de causa e efeito entre o fenômeno investigado e suas consequências $^{16,17}$. A pesquisa foi realizada na Marisol Indústria do Vestuário Ltda., empresa do setor têxtil, uma das maiores indústrias nacionais no segmento de vestuário, sede de Jaraguá do Sul (SC).

O projeto foi aprovado pelo Comitê de Ética em Pesquisa da Universidade do Estado de Santa Catarina (no 83/2011). A amostra foi não probabilística e intencional, já que a escolha do setor produtivo se deu com base nas decisões do comitê de ergonomia da empresa.

$\mathrm{Na}$ busca da prevenção de doenças musculoesqueléticas, a indústria realizou análise ergonômica, e elaborou e executou o plano de adequações dos postos de trabalho. As implementações ocorreram inicialmente nos setores de confecção e dobração da empresa, escolhidos para fazer parte deste estudo quando o comitê de ergonômica da empresa observou a necessidade de os trabalhadores se 
conscientizarem da importância de utilizar os recursos ergonômicos implantados, visto que muitas vezes a equipe que fazia a blitz postural ou a análise ergonômica relatava a não utilização ou o uso inadequado dos recursos disponíveis.

Fizeram parte da população do estudo 400 trabalhadores, sendo que a amostra foi composta por 328 destes. Os critérios da seleção da amostra foram os seguintes: estar ativo no cadastro da empresa durante o período das intervenções e assinar o termo de consentimento livre e esclarecido, dispondo-se a participar das intervenções de ergonomia de conscientização. A perda amostral de 72 trabalhadores foi devido a demissões, mudança de setor ou afastamento por doença ou gestação.

Para testar a metodologia de ergonomia de conscientização, seguiram-se as etapas: pré-avaliação, sensibilização, intervenções e avaliação final.

A pré-avaliação do nível de conhecimento dos trabalhadores foi feita através de um questionário que constava de nove perguntas fechadas, quatro alternativas objetivas e suporte de ilustrações para esclarecimento de cada opção. Assim como as intervenções, o questionário foi construído por uma equipe interdisciplinar (engenheiro, técnico em segurança, fisioterapeuta, educador físico e psicóloga, todos com experiência na área de ergonomia) e foi realizada validação de face $(0,88)$ e clareza $(0,98)^{18,19}$.

A Questão 1 (Q1) abordou o tema Visão, a questão 2 (Q2), o tema Audição, as questões 3 (Q3), 5 (Q5), 6 (Q6) e 7 (Q7), o tema Coluna Vertebral, as questões 4 (Q4) e 8 (Q8), o tema Membros Superiores, e a questão 9 (Q9) abordou o tema Membros Inferiores. Especificamente Q1 questionava aspectos de cuidado com a visão; Q2, cuidados com a audição; Q3, cuidados com a postura ao pegar objeto no chão; $\mathrm{Q} 4$, cuidados com a postura ao pegar objeto no alto; Q5, cuidados com a postura na posição sentada; Q6, cuidado com a postura ao dormir; Q7, cuidado com a postura ao levantar; Q8, cuidado ao carregar objetos; Q9, cuidado por manter-se sentado por alguns instantes no chão.

O questionário ainda contou com duas questões descritivas sobre se os trabalhadores achavam que o conhecimento adquirido nas intervenções do projeto os havia ajudado a melhorar a forma com que exerciam suas atividades no trabalho e em casa. A segunda questão buscava informações sobre melhorias que poderiam ser feitas nas intervenções do projeto. $\mathrm{O}$ questionário foi aplicado antes e após as intervenções, sendo que as duas questões descritivas foram respondidas apenas na segunda avaliação.
Se o trabalhador acertasse todas as questões fechadas (de Q1 a Q9), obteria nove, a nota máxima. Para análise dos dados, além da pontuação total, também foi considerada a porcentagem de acertos dos trabalhadores em cada uma das questões.

Foi feita uma sensibilização, com teatro e dinâmica de desafio, para informar os objetivos e características do projeto. $\mathrm{Na}$ sequência, foram realizadas 13 intervenções educativas com duração de 20 minutos, sobre os temas e respectivos recursos: Corpo Humano (jogos); Visão (atividade experimental e jogo desafio obscuro); Audição (visita à orelha inflável e jogo da intensidade dos sons); Movimento Humano, Coluna Vertebral (protótipos e jogo da coluna); Membros Superiores (Vídeo e atividade com boneco de madeira); e Membros Inferiores (Vídeo e jogo postura real). As intervenções ocorreram de agosto a dezembro de 2012.

Em relação aos temas, focaram-se as posturas em geral e medidas de proteção individual, enfatizando a necessidade do trabalhador ter autocuidado com os movimentos, independente do contexto em que estivesse.

Todas as atividades realizadas tiveram o diferencial: intervenções breves, vivenciais, com recursos pedagógicos inovadores e linguagem acessível. O trabalhador foi agente ativo no processo de construção do conhecimento.

A análise dos resultados foi realizada por meio do teste $t$ de Student para amostras dependentes (pareadas). O nível de significância definido para o presente estudo foi de 0,05 (5\%). Para a análise estatística, foi utilizado o software SPSS 19.0 para Windows.

Já na análise dos dados qualitativos, foi realizada uma análise de conteúdo e significado ${ }^{20}$. Em posse das categorias, foi realizada a codificação do material reunido e verificada a frequência das informações. As categorias obtidas após a codificação foram: (A) melhora ao realizar tarefas no trabalho e/ou em casa; (B) apropriação de conhecimento; (C) melhora nos cuidados com saúde; (D) melhora nos cuidados com postura; (E) mudança de hábito; (F) outros (diminuição de dores corporais, melhora na qualidade de vida, disseminação do conhecimento adquirido).

\section{RESULTADOS}

Com relação às características sociodemográficas da amostra, houve predominância de pessoas do sexo 
feminino ( $\mathrm{n}=326,99 \%)$, e a idade dos indivíduos variou entre 17 e 44 anos, média de 33,94ะ9,15.

Os resultados indicaram que houve impacto da Metodologia de Ergonomia de conscientização no conhecimento dos trabalhadores, com aumento significativo na pontuação das questões: Q3; Q6; Q7; Q9 e na pontuação total do questionário (Tabela 1).

Observando as questões de acordo com o tema a que se referia, constatou-se que ocorreu aumento do número de acertos nas questões relacionadas ao tema Coluna Vertebral $(4,39 \pm 0,79$ para 4,88 $\pm 0,34 ; p<0,001)$ e Membros Inferiores $(0,74 \pm 0,44$ para $0,93 \pm 0,26 ; p<0,001)$.

Ao serem questionados sobre se o conhecimento adquirido no projeto ajudou a melhorar a forma com que exercem as atividades, 10 trabalhadores não responderam a pergunta e 318 responderam que sim. Além disso, 233 descreveram em que aspectos esse conhecimento impactou em sua vida (Figura 1).

\section{DISCUSSÃO}

Os resultados observados demonstraram que houve um aumento significativo da porcentagem de acertos das questões do questionário (Tabela 1), confirmando que os trabalhadores aumentaram seu nível de conhecimento em relação a segurança e saúde.

Esses resultados indicam a importância do impacto da Metodologia de Ergonomia de conscientização no conhecimento dos trabalhadores, uma vez que, segundo pesquisadores ${ }^{21,22}$, o conhecimento adquirido pode levar à mudança de hábitos e, como consequência, à melhora na qualidade de vida devido à prevenção de lesões e acidentes.

As questões nas quais foram identificados ganhos significativos no nível de conhecimento tratavam dos temas: pegar objeto no chão, dormir, levantar, sentar no chão. As questões em que não se observou impacto significativo tratavam dos temas: cuidados com a visão, cuidados com a audição, cuidados com a postura ao sentar e ao pegar objetos no alto. Possivelmente, o impacto nas questões não foi tão expressivo pelo fato da empresa ter realizado, em outros momentos, ações educativas que possam ter abordado a temática, proporcionando um conhecimento prévio aos trabalhadores.

Outra possibilidade que pode ter interferido nesse impacto é o próprio desinteresse pessoal do trabalhador por um tema, por não estar exposto a esse risco no ambiente de trabalho - como exemplo, exposição sonora. É possível que o efeito dessa intervenção em setores nos quais o cuidado com a audição é necessário seja mais significativo.

Além do impacto no conhecimento, os trabalhadores demonstraram melhoras em outros aspectos, como a forma com que utilizam seu corpo nas atividades laborais e de vida diária, procurando realizá-las de forma mais correta. Relataram também melhora na qualidade de vida, redução de dor, entre outros impactos positivos. Achados semelhantes aos encontrados neste estudo foram encontrados por demais pesquisadores ${ }^{21-23}$.

Porém, é importante ressaltar que os programas descritos pelos pesquisadores diferenciam-se da metodologia de ergonomia de conscientização, visto que contavam com exercícios físicos e sessões de relaxamento, tendo o aspecto educacional como complementar, o que não possibilita uma avaliação específica do impacto das intervenções educacionais.

Pesquisadores ${ }^{24-26}$ relatam programas do método Back School (Escola da Postura). Nos estudos relacionados,

Tabela 1. Porcentagem de acertos em relação às questões do questionário sobre saúde e segurança no trabalho antes e após as intervenções de ergonomia de conscientização

$\begin{array}{lccc}\text { Questões } & \begin{array}{c}\text { Acertos } \\ \text { pré-intervenções }\end{array} & \begin{array}{c}\text { Acertos } \\ \text { pós-intervenções }\end{array} & \begin{array}{c}\text { Teste } t \\ \text { dependente (Valor p) }\end{array} \\ \text { Q1 } & 0,99 \pm 0,08 & 0,99 \pm 0,10 & 0,665 \\ \text { Q2 } & 0,98 \pm 0,12 & 0,99 \pm 0,08 & 0,257 \\ \text { Q3 } & 0,77 \pm 0,42 & 0,96 \pm 0,19 & <0,001^{*} \\ \text { Q4 } & 0,99 \pm 0,08 & 1,00 \pm 0,00 & 0,158 \\ \text { Q5 } & 0,99 \pm 0,08 & 1,00 \pm 0,00 & 0,158 \\ \text { Q6 } & 0,91 \pm 0,28 & 0,99 \pm 0,11 & <0,001^{*} \\ \text { Q7 } & 0,72 \pm 0,45 & 0,93 \pm 0,26 & <0,001^{*} \\ \text { Q8 } & 0,97 \pm 0,18 & 0,97 \pm 0,18 & 1,000 \\ \text { Q9 } & 0,74 \pm 0,44 & 0,93 \pm 0,26 & <0,001^{*} \\ \text { Total } & 8,07 \pm 1,03 & 8,76 \pm 0,50 & <0,001^{*}\end{array}$

*diferença significativa entre as porcentagens de acertos nas questões antes e após as intervenções $(p<0,001)$

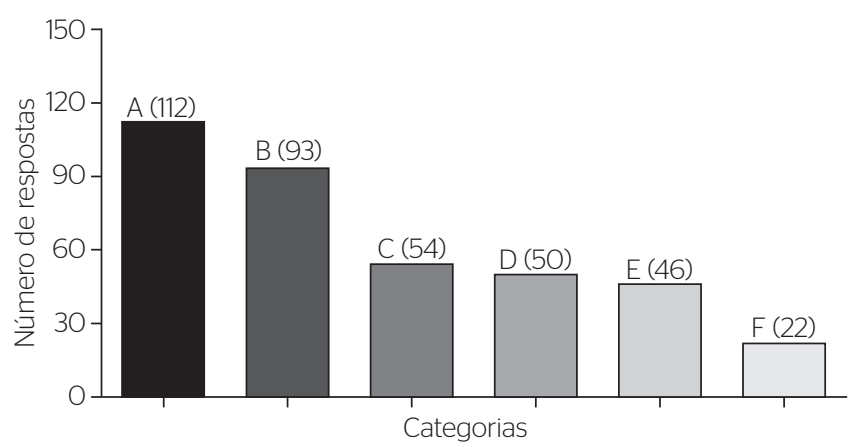

Figura 1. Resultados da resposta à pergunta: Você acha que o conhecimento adquirido no Projeto Ergonomia de Conscientização ajudou a melhorar a forma como você exerce as suas atividades no trabalho e em casa? (A) Melhora ao realizar tarefas no trabalho e/ou em casa; (B) apropriação de conhecimento; (C) melhora nos cuidados com saúde; (D) melhora nos cuidados com postura; (E) mudança de hábito; (F) outros (diminuição de dores corporais, melhora na qualidade de vida, disseminação do conhecimento adquirido) 
observa-se o fato desta ser voltada para pessoas com histórico de dor em coluna e constar de aulas teóricas complementadas por exercícios físicos. Assim como no presente estudo, os autores observaram impacto positivo do programa em seus objetivos, que, no caso, foram sensação de bem-estar, melhora da mobilidade da coluna e melhora da capacidade funcional. Heymans et al. ${ }^{27}$, no entanto, relatam que a maior parte dos estudos relacionados ao método Escola de Postura apresentou resultados positivos em relação à redução da dor e melhora de desempenho funcional quando comparado com outros programas que contavam apenas com exercícios, o que leva a crer que esse resultado deve estar relacionado ao fato do método considerar o indivíduo ativo e participante do processo de saúde.

Já os estudos de Lim et al..$^{28}$ e Garmer, Sperling e Forsberg ${ }^{14}$ constaram de intervenções essencialmente educacionais. A metodologia de ambos os estudos assemelha-se à metodologia proposta por proporcionar experiências práticas de aprendizado que geraram impacto positivo. Lim et al. ${ }^{28}$ analisaram o impacto de um programa de intervenções de ergonomia de conscientização na prevenção de lesões por esforço repetitivo, constatando redução de dor corporal e redução de 38,1\% na probabilidade de ocorrência de lesões no grupo experimental. Garmer, Sperling e Forsberg ${ }^{14}$ se propuseram a trabalhar com a escolha reflexiva dos trabalhadores em relação à escolha de ferramentas manuais, denominando o programa de "aprender fazendo".

Nogueira e Navega ${ }^{29}$ mencionam que os impactos de programas de educação em saúde são extremamente relevantes para a promoção da saúde e melhora na qualidade de vida. Para realizar educação em segurança e saúde de forma diferenciada, a metodologia de ergonomia de conscientização tratou de conteúdos técnicos com intervenções breves, interativas e lúdicas, com conteúdos que retratavam a realidade dos trabalhadores. A metodologia supriu uma necessidade citada na literatura, que é a dos profissionais da saúde contemplarem a realidade em que os indivíduos estão inseridos e substituírem as formas tradicionais de transmissão do conhecimento por estratégias interativas que tornaram o conhecimento transmitido significativo para o receptor ${ }^{13,28,30,31}$.

Weintraub, Hawlitschek e João ${ }^{31}$ enfatizam que, para um aprendizado mais eficaz, é necessário mobilizar o indivíduo para o conhecimento, levando em conta a construção ativa deste e proporcionando a sua síntese. Recursos pedagógicos, tais como jogos, proporcionam a fixação de conceitos e o aprendizado dinâmico devido ao uso da animação e de imagens, o que atrai mais a atenção dos indivíduos. A retenção do conhecimento passa de $20 \%$ em uma exposição com uso de recursos multimídia para $75 \%$ em uma atividade voltada para a prática.

Portanto, a efetividade dos programas de Ergonomia está intimamente atrelada não somente à presença de um ambiente de trabalho seguro, mas igualmente à presença de um público interno informado, capaz de aproveitar suas potencialidades e conhecer suas restrições. Tal resultado conduz ao pensamento de que o processo educativo tem papel determinante na melhoria das condições ergonômicas, haja vista que muitas vezes a empresa pode investir na melhoria das condições do ambiente de trabalho, mas o trabalhador não está habilitado para lidar com as melhorias propostas. $\mathrm{O}$ investimento de uma empresa pode ser posto em risco pelo fato do trabalhador desconhecer o funcionamento e os limites de seu próprio corpo.

Por último, cabe destacar que, ao mobilizar e sensibilizar uma pequena parcela da população para problemas de saúde, como as lesões musculoesqueléticas, dá-se o primeiro passo para pequenas modificações que gradativamente desencadearão novas ações que se refletirão no todo ${ }^{12}$.

\section{CONCLUSÃO}

Os resultados da utilização da Metodologia de Ergonomia de Conscientização apontaram a efetividade da mesma quanto ao nível de conhecimento. Conclui-se que informar o trabalhador da indústria sobre segurança e saúde, ergonomia e consciência corporal, com suporte de recursos didáticos interativos e lúdicos que contemplam a realidade do indivíduo, pode ser uma alternativa promissora na prevenção de doenças ocupacionais, visto que é necessário sensibilizá-los para a questão do autocuidado dentro e fora do ambiente de trabalho.

\section{REFERÊNCIAS}

1. Schubert B. Problemas actuales del seguro obligatorio de accidentes a escala mundial. Associación Internacional de la Seguridad Social. Estocolmo: 27a Asamblea General, 2001.

2. Mossink JCM. Prevención de trastornos musculoesqueléticos en el lugar de trabajo. Organización Mundial de La Salud, 5; 2004. 
3. Cunha JB, Blank VLG, Boing AF. Tendência temporal de afastamento do trabalho em servidores públicos (1995-2005). Rev Bras Epidemiol. 2009:12(2):226-36

4. Almeida LB, Bachur JÁ, Quemelo PRV. Análise ergonômica do setor de prensados para a produção de solados em uma empresa calçadista da cidade de Franca-SP. Investigação. 2010;10:69-73.

5. Brasil. Ministério da Saúde. Doenças relacionadas ao trabalho: Manual de procedimentos para os serviços de saúde. Brasília: Ministério da Saúde, 114(A); 2001.

6. Smedley J, Trevelyan F, Inskip H, Buckle P, Cooper C, Coggon D. Impact of ergonomic intervention on back pain among nurses. Scand J Work Environ Health. 2003;29(2):117-23.

7. Hartvigsen J, Lauritzen S, Lings S, Lauritzen T. Intensive education combined with low tech ergonomic intervention does not prevent low back pain in nurses. Occup Environ Med. 2005;62(1):13-7.

8. Hakala PT, Saarni LA, Ketola RL, Rahkola ET, Salminen JJ, Rimpelä AH. Computer-associated health complains and sources of ergonomic introductions in computer-related issues among Finnish adolescents: a cross-sectional study. BMC Public Health. 2010;10(1):11.

9. Haukka E, Pehkonen I, Leino-Arjas P, Viikari-Juntura E, Takala EP, Malmivaara A, et al. Effect of a participatory ergonomics intervention on psychosocial factors at work in a randomized controlled trial. Occup Environ Med. 2010;67(3):170-7.

10. Bom Sucesso EP. Trabalho e Qualidade de Vida. Rio de Janeiro: Qualitymark/Dunya; 1997

11. Deliberato PCP. Fisioterapia Preventiva: fundamentos e aplicações. São Paulo: Manole; 2002

12. Lida I. Ergonomia: Projeto e Produção. São Paulo: Blucher; 2005.

13. Abrantes AF. Atualidades em ergonomia: logística, movimentação de materiais, engenharia industrial, escritórios. São Paulo: IMAM; 2004.

14. Garmer K, Sperling L, Forsberg A. A hand-ergonomics training kit: development and evaluation of a package to support improved awareness and critical thinking. Appl Ergon. 2002;33(1):39-49.

15. Thornton LJ, Stuart-Buttle C, Wyszynski TC, Wilson ER. Physical and psychosocial stress exposures in US dental schools: the need for expanded ergonomics training. Appl Ergon. 2004;35(2):153-57.

16. Gaya A. Ciências do movimento humano: introdução à metodologia de pesquisa. Porto Alegre: ArtMed; 2008.

17. Minayo MCS. O desafio do conhecimento: pesquisa qualitativa em saúde. 7. ed. São Paulo: Hucitec; 2000.

18. Silveira FL. Validação de instrumentos de medida aplicados à pesquisa em ensino de Física. Porto Alegre: Edipucrs; 1993.
19. Rothstein JR, Tamborindeguy AC. Validação de Clareza de um questionário sobre saúde e segurança no trabalho. Anais do XIX Congresso Brasileiro de Fisioterapia. Florianópolis; 2011.

20. Turato ER. Tratado da Metodologia da Pesquisa Clínica-Qualitativa. Petrópolis: Vozes; 2003.

21. Garcia AN, Gondo FLB, Costa RA, Cyrillo FN, Costa LOP. Efeitos de duas intervenções fisioterapêuticas em pacientes com dor lombar crônica não específica: viabilidade de um estudo controlado aletorizado. Rev Bras Fisioter. 2011;15(5):420-7.

22. Pinafo E, Nunes EFPA, González AD, Garanhani ML. Relações entre concepções e práticas de educação em saúde na visão de uma equipe de saúde da família. Trab Educ Saúde. 2011;9(2):201-21.

23. Andrade SC, Araujo AGR, Vilar JP. A Escola de Postura. Revisão história e suas aplicações na lombalgia crônica. Rev Bras Reumatol. 2005:45(4):224-8.

24. Alexandre NMC, Moraes MAA, Corrêa Filho HR, Jorge SA. Avaliação de um programa para reduzir dores nas costas em trabalhadores de enfermagem de um Hospital Universitário. Rev Saude Publica. 2001;35(4):356-61.

25. Kim P, Hayden JA, Mior AS. The cost-effectiveness of a back education program for firefighters: a case study. J Can Chiropr Assoc. 2004:48(1):13-9.

26. Tsukimoto GR, Riberto $M$, Brito CA, Battistella LR. Avaliação longitudinal da Escola de Postura para dor lombar crônica através da aplicação dos questionários Roland Morris e Short Form Health Survey (SF-36). Acta Fisiatr. 2006;13(2):63-9.

27. Heymans MW, van Tulder MW, Esmail R, Bombardier C, Koes BW. Back schools for nonspecific low back pain: a systematic review within the framework of the Cochrane Collaboration Back Review Croup. Spine (Phila Pa 1976). 2005;30(19):2153:63.

28. Lim HJ, Black TR, Shah SM, Sarker S, Metcalfe J. Evaluating repeated patient handling injuries following the implementation of a multifactor ergonomic intervention program among health care workers. J Safety Res. 2011;42(3):185-91.

29. Nogueira HC; Navega MT. Influência da escola de postura na qualidade de vida, capacidade funcional, intensidade de dor e flexibilidade de trabalhadores administrativos. Fisioter Pesq. 2011;18(4)353-8.

30. Stroschein KA, Zocche DAA. Educação permanente nos serviços de saúde: um estudo sobre as experiências realizadas no Brasil. Trab Educ Saúde. 2011;9(3):505-19.

31. Weintraub M, Hawlitschek P, João SMA. Jogo educacional sobre avaliação em fisioterapia: uma nova abordagem acadêmica. Fisioter Pesq. 2011:18(3):280-6. 


\section{Fisioter Pesq. 2013;20(1):11-16}

Artigo: Impacto de uma metodologia interativa de ergonomia de conscientização

Página 11, Onde se lê:

Estudo desenvolvido na Universidade do Estado de Santa Catarina - Florianópolis (SC), Brasil.

Leia-se:

Estudo desenvolvido pelo Serviço Social da Indústria de Santa Catarina - SESI (DR) - Florianópolis (SC), Brasil. 